\title{
Rank two jump loci for solvmanifolds and Lie algebras
}

\author{
By Ştefan Papadima and Laurenţiu Paunescu
}

(Received Feb. 15, 2016)

(Revised Sep. 19, 2016)

\begin{abstract}
We consider representation varieties in $S L_{2}$ for lattices in solvable Lie groups, and representation varieties in $\mathfrak{s l}_{2}$ for finite-dimensional Lie algebras. Inside them, we examine depth 1 characteristic varieties for solvmanifolds, respectively resonance varieties for cochain Differential Graded Algebras of Lie algebras. We prove a general result that leads, in both cases, to the complete description of the analytic germs at the origin, for the corresponding embedded rank 2 jump loci.
\end{abstract}

\section{Introduction and statement of results.}

Jump loci are basic objects in geometry, topology and group theory. They appear in the following way. Let $M$ be a connected, compact differentiable manifold (up to homotopy), with fundamental group $\pi$. Let $\iota: \mathbb{G} \rightarrow G L(V)$ be an algebraic representation of complex linear algebraic groups. The representation variety $\operatorname{Hom}(\pi, \mathbb{G})$ is an affine variety with distinguished basepoint 1 , the trivial group homomorphism. It encodes the representation theory of $\pi$ into $\mathbb{G}$. Furthermore, it comes equipped with a collection of closed subvarieties: the characteristic varieties (or jump loci) in degree $i$ and depth $r$

$$
\mathscr{V}_{r}^{i}(M, \iota)=\left\{\rho \in \operatorname{Hom}(\pi, \mathbb{G}) \mid \operatorname{dim} H^{i}\left(M,{ }_{\iota \rho} V\right) \geq r\right\},
$$

which stratify the local systems on $M$ with typical fiber $V$ that factor through $\iota$, according to the dimension of the corresponding twisted cohomology of $M$. This rich structure contains valuable information on the geometry and topology of $M$.

The rank one case (when $\iota=\mathrm{id}_{\mathbb{C} \times}$ ) received a lot of attention. The corresponding characteristic varieties, denoted simply by $\mathscr{V}_{r}^{i}(M)$, are the topological counterpart, for $r=1$, of the loci defined in algebraic geometry by Green and Lazarsfeld in [9][10], when $M$ is a complex projective manifold. Results of Arapura [1], further refined in [6], show how the regular maps from $M$ onto curves of general type may be recovered from the geometry of the variety $\mathscr{V}_{1}^{1}(M)$, when $M$ is supposed merely quasi-projective. In topology, it is known that the rank 1 jump loci $\mathscr{V}_{1}^{i}(M)$ control delicate finiteness properties of Alexander-type invariants of $M$. This fact was used in [4][3] to obtain significant results on certain important subgroups of mapping class groups of closed Riemann surfaces.

2010 Mathematics Subject Classification. Primary 55N25; Secondary 17B56, 20J06, 22 E40.

Key Words and Phrases. representation variety, characteristic variety, resonance variety, analytic germ, solvmanifold, Lie algebra.

The first author partially supported by PN-II-ID-PCE-2011-3-0288, grant 132/05.10.2011.

The first author, Prof. Papadima, passed away before the publication of this paper. 
The rank two case (when $\mathbb{G}$ is $S L_{2}(\mathbb{C})$ or $P S L_{2}(\mathbb{C})$ ) is considerably more difficult. Indeed, the universality theorem of Kapovich and Millson [13] shows that the varieties $\operatorname{Hom}\left(\pi, P S L_{2}(\mathbb{C})\right)$ have arbitrarily bad singularities away from 1 , when $\pi$ runs through the family of Artin groups. At the same time, the large number of applications of this case makes it very interesting. For example, a recent result from [23] establishes a connexion between the rank 2 case and a difficult open problem about the monodromy action on the Milnor fiber homology of a complex hyperplane arrangement.

In this note, we approach analytic germs at 1 of representation varieties and jump loci through the prism of the main result from [5]. The idea is to replace $M$ by a model $A=\left(A^{\bullet}, d\right)$ : a connected, finite-dimensional Commutative Differential Graded Algebra (for short, a cdga) having the same Sullivan minimal model [26] as the complex de Rham cdga of $M$. Denote by $\theta: \mathfrak{g} \rightarrow \mathfrak{g l}(V)$ the tangent map at 1 of $\iota$. Replace the $\mathbb{G}$-representation variety of $\pi$ by the affine variety of $\mathfrak{g}$-valued flat connections on $A$, $\mathscr{F}(A, \mathfrak{g}) \subseteq A^{1} \otimes \mathfrak{g}$, with natural basepoint 0 . For $\omega \in \mathscr{F}(A, \mathfrak{g})$, consider the cochain complex $\left(A^{\bullet} \otimes V, d_{\omega}\right)$, where $d_{\omega}$ is the associated algebraic covariant derivative. Replace $\mathscr{V}_{r}^{i}(M, \iota)$ in (1) by the resonance varieties $\mathscr{R}_{r}^{i}(A, \theta)$ defined in (2). These Zariski closed subvarieties of $\mathscr{F}(A, \mathfrak{g})$ record how $\operatorname{dim} H^{i}\left(A \otimes V, d_{\omega}\right)$ jumps, for $\omega \in \mathscr{F}(A, \mathfrak{g})$. See Section 2 for more details.

In what follows, we will denote by $\mathcal{X}_{(x)}$ the analytic germ at $x$ of an affine variety $\mathcal{X}$. Theorem B from [5] establishes an isomorphism $\operatorname{Hom}(\pi, \mathbb{G})_{(1)} \simeq \mathscr{F}(A, \mathfrak{g})_{(0)}$ that identifies $\mathscr{V}_{r}^{i}(M, \iota)_{(1)}$ with $\mathscr{R}_{r}^{i}(A, \theta)_{(0)}$ for all $i, r$, when $A$ models $M$. We are going to examine topological and algebraic Green-Lazarsfeld loci, corresponding to $r=1$. In the rank 1 case, we will drop $\iota$ and $\theta$ from notation.

To state our first main result, we need to recall two constructions from [18] (see also Section 2). The Zariski closed subset $\mathscr{F}^{1}(A, \mathfrak{g}) \subseteq \mathscr{F}(A, \mathfrak{g})$ consists of those flat connections of the form $\omega=\eta \otimes g$ with $d \eta=0$. The additional condition $\operatorname{det} \theta(g)=0$ defines the Zariski closed subset $\Pi(A, \theta) \subseteq \mathscr{F}^{1}(A, \mathfrak{g})$.

TheOREM 1.1 (Corollary 2.5). Let $A=\left(A^{\bullet}, d\right)$ be a connected, finite-dimensional cdga over $\mathbb{C}$. Let $\theta: \mathfrak{g} \rightarrow \mathfrak{g l}(V)$ be a finite-dimensional representation of a finitedimensional Lie algebra $\mathfrak{g}$, with $V \neq 0$. Assume that 0 is an isolated point of $\bigcup_{i \geq 0} \mathscr{R}_{1}^{i}(A)$, and $\mathscr{F}(A, \mathfrak{g})_{(0)}=\mathscr{F}^{1}(A, \mathfrak{g})_{(0)}$. Then $\mathscr{R}_{1}^{i}(A, \theta)_{(0)}=\Pi(A, \theta)_{(0)}$, for all $i$.

In Section 3, we apply this general result to a discrete, co-compact subgroup $\Gamma$ of a 1-connected solvable real Lie group $S$ and the associated aspherical solvmanifold $M=S / \Gamma$. It leads to the following complete description of embedded germs at 1 of rank two topological Green-Lazarsfeld loci, for solvmanifolds. (In what follows, we denote by $\mathbb{P}(H)$ the projective space of a vector space, and by $V(f)$ the zero set of a polynomial.)

Theorem 1.2 (Theorem 3.3). Let $M=S / \Gamma$ be a solvmanifold and let $\iota: \mathbb{G} \rightarrow$ $G L(V)$ be a rational representation of a complex, semisimple linear algebraic group of rank 1 , with tangent map $\theta: \mathfrak{s l}_{2} \rightarrow \mathfrak{g l}(V)$. Then the germ at 1 of $\operatorname{Hom}(\Gamma, \mathbb{G})$ is isomorphic to the germ at 0 of the cone on $\mathbb{P}\left(H^{1}(M)\right) \times \mathbb{P}\left(\mathfrak{s l}_{2}\right)$, and the embedded topological GreenLazarsfeld germs are given by 


$$
\mathscr{V}_{1}^{i}(M, \iota)_{(1)}= \begin{cases}\emptyset & \text { if } H^{i}(M)=0 \\ \operatorname{cone}\left(\mathbb{P}\left(H^{1}(M)\right) \times V(\operatorname{det} \circ \theta)\right)_{(0)} & \text { otherwise. }\end{cases}
$$

This extends the computation done in [18] for nilmanifolds, by replacing Nomizu's model [21] of such a manifold with a model found by Kasuya [14] for an arbitrary solvmanifold. As explained in [25], lattices in solvable Lie groups are much more complicated than those in nilpotent Lie groups. Nevertheless, Theorem 1.1 makes things work well. Note that the first assumption from Theorem 1.1 (on rank one resonance) also appears in Theorem 1 from [7]: it is equivalent to the fact that all completed Alexander invariants of $M$ are finite-dimensional, for an arbitrary compact manifold $M$ modeled by $A$. To check it for solvmanifolds, we prove in Theorem 3.5 a result of independent interest: each rank one characteristic variety $\mathscr{V}_{1}^{i}(M)$ is finite, when $M$ is the classifying space of a virtually polycyclic group.

Next, we recall that Nomizu's model for the nilmanifold $M=S / \Gamma$ is the ChevalleyEilenberg cochain cdga, $\mathcal{C} \bullet(\mathfrak{s} \otimes \mathbb{C})$, of the nilpotent Lie algebra $\mathfrak{s} \otimes \mathbb{C}$, where $\mathfrak{s}$ is the Lie algebra of $S$. In Section 4, we apply Theorem 1.1 to another large class of examples: cochain cdga's of arbitrary finite-dimensional Lie algebras. Denoting by $H^{\bullet}(\mathfrak{h})$ untwisted Lie algebra cohomology, we obtain in Theorem 4.8 the following complete description of embedded germs at 0 of rank two algebraic Green-Lazarsfeld loci, for this class.

TheOrem 1.3 (Theorem 4.8). Let $\mathfrak{h}$ be a finite-dimensional complex Lie algebra and let $\theta: \mathfrak{s l}_{2} \rightarrow \mathfrak{g l}(V)$ be a finite-dimensional Lie representation with $V \neq 0$. Then $\mathscr{F}\left(\mathcal{C}^{\bullet} \mathfrak{h}, \mathfrak{s l}_{2}\right)_{(0)}$ is equal to $\{0\}$ when $H^{1}(\mathfrak{h})=0$, and otherwise is isomorphic to cone $\left(\mathbb{P}\left(H^{1}(\mathfrak{h})\right) \times \mathbb{P}\left(\mathfrak{s l}_{2}\right)\right)_{(0)}$. In the first case, $\mathscr{R}_{1}^{i}\left(\mathcal{C}^{\bullet} \mathfrak{h}, \theta\right)_{(0)}$ is empty or equal to $\{0\}$, depending on whether $H^{i}(\mathfrak{h})$ vanishes or not. In the second case, the embedded germs of depth 1 resonance varieties are given by

$$
\mathscr{R}_{1}^{i}\left(\mathcal{C}^{\bullet} \mathfrak{h}, \theta\right)_{(0)}= \begin{cases}\emptyset & \text { if } H^{i}(\mathfrak{h})=0, \\ \operatorname{cone}\left(\mathbb{P}\left(H^{1}(\mathfrak{h})\right) \times V(\operatorname{det} \circ \theta)\right)_{(0)} & \text { otherwise. }\end{cases}
$$

When $A^{\bullet}=\mathcal{C}^{\bullet} \mathfrak{h}$, the analysis of the second hypothesis from Theorem 1.1 (on flat connections) turns out to be the more difficult part. We know from $[\mathbf{1 8}]$ that $\mathscr{F}\left(A, \mathfrak{s l}_{2}\right)=$ $\mathscr{F}^{1}\left(A, \mathfrak{s l}_{2}\right)$, when $\mathfrak{h}$ is nilpotent. We first extend this property to the solvable case, where we prove that $\mathscr{F}\left(\mathcal{C}^{\bullet} \mathfrak{h}, \mathfrak{s l}_{2}\right)_{(0)}=\mathscr{F}^{1}\left(\mathcal{C}^{\bullet} \mathfrak{h}, \mathfrak{s l}_{2}\right)_{(0)}$. Along the way, we show in Lemma $4.3(2)$ that the global property $\mathscr{F}\left(\mathcal{C}^{\bullet} \mathfrak{h}, \mathfrak{s l}_{2}\right)=\mathscr{F}^{1}\left(\mathcal{C}^{\bullet} \mathfrak{h}, \mathfrak{s l}_{2}\right)$ actually characterizes the nilpotence of $\mathfrak{h}$, within a certain metabelian class. Finally, we associate to an arbitrary finitedimensional Lie algebra $\mathfrak{h}$ a solvable Lie algebra $\widetilde{\mathfrak{s}}$, and we prove in Proposition 4.6 that, for any finite-dimensional Lie algebra $\mathfrak{k}$, there is a natural analytic isomorphism, $\mathscr{F}\left(\mathcal{C}^{\bullet} \mathfrak{h}, \mathfrak{k}\right)_{(0)} \simeq \mathscr{F}\left(\mathcal{C}^{\bullet} \widetilde{\mathfrak{s}}, \mathfrak{k}\right)_{(0)}$. This leads to the equality $\mathscr{F}\left(\mathcal{C}^{\bullet} \mathfrak{h}, \mathfrak{s l}_{2}\right)_{(0)}=\mathscr{F}^{1}\left(\mathcal{C}^{\bullet} \mathfrak{h}, \mathfrak{s l}_{2}\right)_{(0)}$, in the general case.

\section{Vanishing rank one resonance.}

We start by isolating a useful property of rank 1 resonance. In the sequel, this will enable us to treat simultaneously the germs at the origin of rank 2 jump loci, for both 
solvmanifolds and Lie algebras.

First, we need to review a couple of basic definitions and facts related to algebraic jump loci, following $[\mathbf{5}]$ and $[\mathbf{1 8}]$. Unless otherwise mentioned, our ground ring will be $\mathbb{C}$.

Let $A=\left(A^{\bullet}, d\right)$ be a cdga, whose defining axioms capture the essential algebraic features of the de Rham algebra of a differentiable manifold. Our standard assumption is that $A$ is finite-dimensional (as a vector space) and connected (i.e., $A^{0}=\mathbb{C} \cdot 1$ ). For a finite-dimensional Lie algebra $\mathfrak{g}$, we denote by $\mathscr{F}(A, \mathfrak{g}) \subseteq A^{1} \otimes \mathfrak{g}$ the variety of $\mathfrak{g}$ valued flat connections on $A$, given by the solutions of the Maurer-Cartan equation, $d \omega+[\omega, \omega] / 2=0$. This construction gives a Zariski closed subset of $A^{1} \otimes \mathfrak{g}$ containing the distinguished point 0 , and is natural in both $A$ and $\mathfrak{g}$. When $\mathfrak{g}$ is abelian, $\mathscr{F}(A, \mathfrak{g})=$ $H^{1}(A) \otimes \mathfrak{g}$, since $A$ is connected. Otherwise, the singularity $\mathscr{F}(A, \mathfrak{g})_{(0)}$ can be pretty complicated. We will denote $\mathscr{F}(A, \mathbb{C})$ simply by $\mathscr{F}(A)$.

Now, let $\theta: \mathfrak{g} \rightarrow \mathfrak{g l}(V)$ be a finite-dimensional Lie representation with $V \neq 0$. For $\omega \in \mathscr{F}(A, \mathfrak{g})$, the covariant derivative $d_{\omega}$ acts on $A^{\bullet} \otimes V$ (via $\theta$ ), in the following way. Write $\omega=\sum_{i} \eta_{i} \otimes g_{i} \in A^{1} \otimes \mathfrak{g}$. Then $d_{\omega}(\eta \otimes v)=d \eta \otimes v+\sum_{i} \eta_{i} \eta \otimes \theta\left(g_{i}\right) v$, for $\eta \otimes v \in A^{\bullet} \otimes V$. The flatness condition implies that $d_{\omega}^{2}=0$. We may thus speak for each degree $i$ about the descending filtration of $\mathscr{F}(A, \mathfrak{g})$ by the depth $r$ resonance varieties

$$
\mathscr{R}_{r}^{i}(A, \theta)=\left\{\omega \in \mathscr{F}(A, \mathfrak{g}) \mid \operatorname{dim} H^{i}\left(A \otimes V, d_{\omega}\right) \geq r\right\},
$$

which are Zariski closed in $\mathscr{F}(A, \mathfrak{g})$. We are going to pay particular attention to the depth 1 resonance varieties, since their germs at 0 are isomorphic to the germs at 1 of the corresponding topological Green-Lazarsfeld loci $\mathscr{V}_{1}^{i}(M, \iota)$, when $A$ models $M$.

The simplest case is the rank one case, corresponding to $\theta=\mathrm{id}_{\mathbb{C}}$, when $\mathscr{R}_{r}^{i}(A, \theta) \subseteq$ $\mathscr{F}(A, \mathbb{C})$ is denoted simply by $\mathscr{R}_{r}^{i}(A) \subseteq \mathscr{F}(A)$, and $\mathscr{F}(A)=H^{1}(A)$. This case is much more studied than the non-abelian situation. Our starting point in this note is to identify properties of rank 1 resonance that give valuable information on higher rank resonance. A simple useful remark in this direction, made in [18], is that

$$
\mathscr{R}_{1}^{i}(A, \theta)_{(0)} \neq \emptyset \Longleftrightarrow \mathscr{R}_{1}^{i}(A)_{(0)} \neq \emptyset \Longleftrightarrow H^{i}(A) \neq 0 \text {. }
$$

More information comes from considering the quadratic map

$$
P: A^{1} \times \mathfrak{g} \longrightarrow A^{1} \otimes \mathfrak{g}, \quad(\eta, g) \mapsto \eta \otimes g,
$$

the induced map $P: H^{1}(A) \times \mathfrak{g} \longrightarrow \mathscr{F}(A, \mathfrak{g})$, and the (essentially) rank one part $\mathscr{F}^{1}(A, \mathfrak{g}):=P\left(H^{1}(A) \times \mathfrak{g}\right)$, which is Zariski closed in $\mathscr{F}(A, \mathfrak{g})$ and contains the origin 0 . Define $\Pi(A, \theta):=P\left(H^{1}(A) \times V(\operatorname{det} \circ \theta)\right)$, where det $: \mathfrak{g l}(V) \rightarrow \mathbb{C}$ is the determinant. Again, $\Pi(A, \theta)$ is Zariski closed in $\mathscr{F}^{1}(A, \mathfrak{g})$ and contains 0 . Moreover, as shown in [18], $H^{i}(A) \neq 0$ implies that

$$
\Pi(A, \theta) \subseteq \mathscr{R}_{1}^{i}(A, \theta) .
$$

This higher rank resonance bound actually follows from a more precise result.

THEOREM 2.1 ([18]). Let $\omega=\eta \otimes g$ with $d \eta=0$ be an arbitrary element of $\mathscr{F}^{1}(A, \mathfrak{g})$. Then $\omega \in \mathscr{R}_{1}^{i}(A, \theta)$ if and only if there is an eigenvalue $\lambda$ of $\theta(g)$ such that 
$\lambda \eta \in \mathscr{R}_{1}^{i}(A)$.

In coordinates, consider the quadratic map

$$
P: \mathbb{C}^{m} \times \mathbb{C}^{n} \longrightarrow \mathbb{C}^{m n} \equiv \mathbb{C}^{m} \otimes \mathbb{C}^{n}, \quad(x, y) \mapsto z,
$$

where $z_{i j}=x_{i} y_{j}$. We denote its image by $\mathscr{F}^{1}(m, n)$. Clearly, $\mathscr{F}^{1}(m, n)=\mathscr{F}^{1}(A, \mathfrak{g})$, when $\operatorname{dim} H^{1}(A)=m$ and $\operatorname{dim} \mathfrak{g}=n$. As is well-known, $\mathscr{F}^{1}(m, n) \simeq \operatorname{cone}\left(\mathbb{P}^{m-1} \times \mathbb{P}^{n-1}\right)$ is Zariski closed in $\mathbb{C}^{m} \otimes \mathbb{C}^{n}$.

Clearly, $P^{-1}(0)=\mathbb{C}^{m} \times 0 \bigcup 0 \times \mathbb{C}^{n}$. To describe the other fibers, let us consider the $\mathbb{C}^{\times}$-action defined by $t \cdot(x, y)=\left(t^{-1} x, t y\right)$, for $t \in \mathbb{C}^{\times}$. Then $P(x, y)=P\left(x^{\prime}, y^{\prime}\right)$ if and only if $(x, y)$ and $\left(x^{\prime}, y^{\prime}\right)$ lie in the same $\mathbb{C}^{\times}$-orbit, when $P(x, y) \neq 0$.

We will use the following nice property of the regular map (6).

Lemma 2.2. A regular function $f$ on $\mathbb{C}^{m} \times \mathbb{C}^{n}$ factors through the surjection $P: \mathbb{C}^{m} \times \mathbb{C}^{n} \rightarrow \mathscr{F}^{1}(m, n)$ if and only if $f$ is $\mathbb{C}^{\times}$-invariant. If this is the case, then the (unique) quotient function $F$ is regular on $\mathscr{F}^{1}(m, n)$.

Proof. If $f$ factors, then clearly $f$ is $\mathbb{C}^{\times}$-invariant, since $P(t \cdot(x, y))=P(x, y)$, for all $t \in \mathbb{C}^{\times}$and $(x, y) \in \mathbb{C}^{m} \times \mathbb{C}^{n}$. Conversely, $\mathbb{C}^{\times}$-invariance means that the polynomial $f$ is of the form $f(x, y)=\sum_{|I|=|J|} c_{I J} x_{I} y_{J}$ where $(I, J) \in \mathbb{N}^{m} \times \mathbb{N}^{n}$. This plainly implies factorization, according to the above description of the fibers of $P$. The second claim follows from the fact that the algebra of $\mathbb{C}^{\times}$-invariant polynomials is generated by the monomials $x_{i} y_{j}$.

Definition 2.3. We say that the cdga $A$ has trivial resonance in degree $i$ if 0 is an isolated point of $\mathscr{R}_{1}^{i}(A)$. In other words, $\mathscr{R}_{1}^{i}(A)=\{0\} \cup \bigcap_{\alpha}\left(V\left(\varphi_{\alpha}\right) \cap H^{1}(A)\right)$, where $\left\{\varphi_{\alpha}\right\}$ are polynomial functions on $A^{1}$ and there is $\varphi_{0}$ such that $\varphi_{0}(0) \neq 0$.

This property has the following significant higher rank consequence.

THEOREm 2.4. If $A$ has trivial resonance in degree $i$, then $\Pi(A, \theta)$ and $\mathscr{R}_{1}^{i}(A, \theta) \cap$ $\mathscr{F}^{1}(A, \mathfrak{g})$ have the same germ at 0 , for any representation $\theta: \mathfrak{g} \rightarrow \mathfrak{g l}(V)$.

Proof. $\quad$ By (3) and (5), we know that $0 \in \Pi(A, \theta) \subseteq \mathscr{R}_{1}^{i}(A, \theta) \cap \mathscr{F}^{1}(A, \mathfrak{g})$. Set $p=\operatorname{dim} V$ and consider the polynomial function on $A^{1} \times \mathbb{C}^{p}, \widetilde{f}(\eta, \lambda)=\prod_{i=1}^{p} \varphi_{0}\left(\lambda_{i} \eta\right)$, where $\varphi_{0}$ is chosen as in Definition 2.3. Since $\tilde{f}$ is symmetric in $\lambda, \tilde{f}(\eta, \lambda)=\bar{f}(\eta, \sigma)$, for some polynomial function $\bar{f}$ on $A^{1} \times \mathbb{C}^{p}$, when $\sigma_{i}$ is the $i$-th elementary symmetric function $\sigma_{i}(\lambda)$, for $i=1, \ldots, p$. Next, write $\operatorname{det}(t \cdot \mathrm{id}-\theta(g))=t^{p}+\sum_{i=1}^{p}(-1)^{i} c_{i} t^{p-i}$, where each $c_{i}$ is a polynomial function in $g \in \mathfrak{g}$. Clearly, $c_{i}(g)=\sigma_{i}(\lambda)$, for all $i$, where $\lambda_{1}, \ldots, \lambda_{p}$ are the eigenvalues of $\theta(g)$. Define the polynomial function $f$ on $A^{1} \times \mathfrak{g}$ by $f(\eta, g)=\bar{f}(\eta, c(g))$. By construction, $f(\eta, g)=\widetilde{f}(\eta, \lambda)$, when $\lambda_{1}, \ldots, \lambda_{p}$ are the eigenvalues of $\theta(g)$.

We may thus apply Lemma 2.2 to the quadratic map (4) and the regular function $f$. Let us check that $f$ factors through $P$. If $\eta=0$ or $g=0$, then clearly $f(\eta, g)=\varphi_{0}(0)^{p}$. It is equally clear that $f\left(t^{-1} \eta, t g\right)=f(\eta, g)$. Hence, we may find a regular function $F$ on $A^{1} \otimes \mathfrak{g}$ such that $F \circ P=f$ and $F(0)=\varphi_{0}(0)^{p} \neq 0$. 
Our claim will follow from the inclusion $\mathscr{R}_{1}^{i}(A, \theta) \cap \mathscr{F}^{1}(A, \mathfrak{g}) \subseteq \Pi(A, \theta) \cup V(F)$. To prove this inclusion, assume that $\omega \in \mathscr{R}_{1}^{i}(A, \theta)$, where $\omega=\eta \otimes g$ with $d \eta=0, \eta \neq 0$ and $\operatorname{det} \theta(g) \neq 0$. We infer from Theorem 2.1 that there is an eigenvalue $\lambda_{j} \neq 0$ of $\theta(g)$ such that $\lambda_{j} \eta \in \mathscr{R}_{1}^{i}(A)$. Therefore, $\varphi_{0}\left(\lambda_{j} \eta\right)=0$, according to Definition 2.3. By the construction of $f, 0=\widetilde{f}(\eta, \lambda)=f(\eta, g)$. Finally, the factorization property of $f$ implies that $f(\eta, g)=F(\omega)$, and we are done.

Under an additional hypothesis, the above result implies that the bound (5) is sharp, at the level of germs at the origin.

Corollary 2.5. If $A$ has trivial resonance in degree $i$ and $\mathscr{F}(A, \mathfrak{g})_{(0)}=$ $\mathscr{F}^{1}(A, \mathfrak{g})_{(0)}$, then $\mathscr{R}_{1}^{i}(A, \theta)_{(0)}=\Pi(A, \theta)_{(0)}$, for any representation $\theta: \mathfrak{g} \rightarrow \mathfrak{g l}(V)$.

The trivial resonance property is also related to delicate finiteness properties of Alexander invariants of spaces [22][7]. For cdga's with $d=0$, it follows from Definition (2) that all resonance varieties $\mathscr{R}_{r}^{i}(A, \theta)$ are homogeneous. In particular, in this case, triviality of resonance in degree $i$ is equivalent to $\mathscr{R}_{1}^{i}(A)=\{0\}$. As shown in [22], examples of this sort abound. More precisely, the cdga's with $d=0$ and fixed Betti numbers $b_{i}=\operatorname{dim} A^{i}$ may be viewed as the points of an affine variety. On this parameter space, triviality of resonance in degree $i$ is a Zariski open condition, when $b_{i}>0$. Moreover, for $i=1$ and $b_{2} \geq 2 b_{1}-3 \geq-1$, this open set is non-void, as follows from Theorem $1.1 \mathrm{in}$ [24]. In the next sections, we will exhibit new classes of examples, with trivial resonance and non-zero differential.

ExAmple 2.6. For $n \geq 2$, let $M=\mathbb{C} \backslash\{n$ points $\}$ be the classifying space of a finitely generated free non-abelian group. It is well-known that in this case $A=$ $\left(H^{\bullet}(M), d=0\right)$ is a model of $M$, and $\mathscr{R}_{1}^{1}(A)=\mathbb{C}^{n}$. Hence, $A$ does not have trivial resonance in degree 1.

This simple example also shows that the second assumption from Corollary 2.5 is not always satisfied. Indeed, for an arbitrary cdga $A=\left(A^{\bullet}, d\right)$ and $\omega=\sum_{i} \eta_{i} \otimes g_{i} \in A^{1} \otimes \mathfrak{g}$, the Maurer-Cartan equation is equivalent to

$$
\sum_{i} d \eta_{i} \otimes g_{i}+\sum_{i<j} \eta_{i} \eta_{j} \otimes\left[g_{i}, g_{j}\right]=0
$$

In our example, $(7)$ shows that $\mathscr{F}(A, \mathfrak{g})=A^{1} \otimes \mathfrak{g}$. When $\operatorname{dim} \mathfrak{g}>1$, it follows that $\mathscr{F}(A, \mathfrak{g})_{(0)} \neq \mathscr{F}^{1}(A, \mathfrak{g})_{(0)}$.

\section{Solvmanifolds.}

In this section, we apply the general theory to solvmanifolds, where it leads to a complete description of germs at 1 for both varieties of $S L_{2}$-representations and topological Green-Lazarsfeld loci. We also show that the rank one topological Green-Lazarsfeld sets of virtually polycyclic groups are finite.

A solv-lattice is a discrete, co-compact subgroup $\Gamma$ of a 1-connected solvable real Lie group $S$, giving rise to the compact, aspherical solvmanifold $M=S / \Gamma$, with fundamental group $\Gamma$. For such a manifold, Kasuya constructed in $[\mathbf{1 4}]$ a connected, finite-dimensional 
cdga model. Since the details are rather complicated, we are going to extract only the properties of the Kasuya model $A$ of $M$ that are relevant to our study, following [14][16].

Let $\mathfrak{n}$ be the nilshadow of the solvable Lie algebra $\mathfrak{s}$ of $S$. The model $A^{\bullet}$ is a sub-cdga of the Chevalley-Eilenberg cochain algebra of the finite-dimensional nilpotent Lie algebra $\mathfrak{n} \otimes \mathbb{C}, B^{\bullet}:=\mathcal{C}^{\bullet}(\mathfrak{n} \otimes \mathbb{C})$. This information is enough to prove that $A$ satisfies the second assumption from Corollary 2.5 globally, for $\mathfrak{g}=\mathfrak{s l}_{2}$.

Lemma 3.1. If $A$ is a Kasuya solvmanifold model and $\mathfrak{g}=\mathfrak{s l}_{2}$, then $\mathscr{F}(A, \mathfrak{g})=$ $\mathscr{F}^{1}(A, \mathfrak{g})$.

Proof. Since $A^{\bullet} \subseteq B^{\bullet}$, equation (7) readily implies that $\mathscr{F}(A, \mathfrak{g})=\mathscr{F}(B, \mathfrak{g}) \cap$ $A^{1} \otimes \mathfrak{g}$, for any $\mathfrak{g}$. Similarly, $\mathscr{F}^{1}(A, \mathfrak{g})=\mathscr{F}^{1}(B, \mathfrak{g}) \cap A^{1} \otimes \mathfrak{g}$. Finally, the nilpotence of $\mathfrak{n} \otimes \mathbb{C}$ implies, for $\mathfrak{g}=\mathfrak{s l}_{2}$, the equality $\mathscr{F}(B, \mathfrak{g})=\mathscr{F}^{1}(B, \mathfrak{g})[\mathbf{1 8}]$. Our claim follows.

To verify triviality of resonance for Kasuya models, we will use a general result from [7], which we now recall. Let $M$ be a connected, finite CW-complex, with fundamental group $\pi$ and universal abelian cover $M^{\mathrm{ab}}$. The action of $\pi_{\mathrm{ab}}$ by deck-transformations makes $H_{\bullet}\left(M^{\mathrm{ab}}\right)$ a graded $\mathbb{C}\left[\pi_{\mathrm{ab}}\right]$-module, with $I$-adic completion denoted $\widehat{H}_{\bullet}\left(M^{\mathrm{ab}}\right)$, where $I$ is the augmentation ideal of the group ring $\mathbb{C}\left[\pi_{\mathrm{ab}}\right]$. Let $A$ be a cdga model of a connected, compact manifold $M$. Then 0 is an isolated point of $\bigcup_{i \geq 0} \mathscr{R}_{1}^{i}(A)$ if and only if the $\mathbb{C}$-vector space $\widehat{H}_{\bullet}\left(M^{\mathrm{ab}}\right)$ is finite-dimensional. When $M=S / \Gamma$ is a solvmanifold, note that $H_{\bullet}\left(M^{\mathrm{ab}}\right)=H_{\bullet}\left(\Gamma^{\prime}\right)$, where $\Gamma^{\prime}$ is the derived subgroup.

Lemma 3.2. All connected covers of a solvmanifold $M=S / \Gamma$ have finite dimensional homology. Moreover, if $H^{i}(M) \neq 0$, then $A$ has trivial resonance in degree $i$, for an arbitrary cdga model $A$ of $M$.

Proof. By the above discussion and (3), it is enough to show that the vector space $H_{\bullet}(G)$ is finite-dimensional, for any subgroup $G \subseteq \Gamma$. This in turn will follow from results in $[\mathbf{2 5}$, Chapters III-IV]. We know that the solv-lattice $\Gamma$ is a polycyclic group, by [25, Proposition 3.7]. Hence, $G$ must be polycyclic [25, Remark 4.2]. According to Theorem 4.28 from [25], $G$ contains as a normal subgroup of finite index a solv-lattice $\Gamma_{0}$, with associated (compact) solvmanifold $M_{0}$. A transfer argument shows then that $H_{\bullet}(G)$ is a quotient of $H_{\bullet}\left(\Gamma_{0}\right)=H_{\bullet}\left(M_{0}\right)$, and we are done.

We may now describe germs at 1 of non-abelian topological Green-Lazarsfeld loci for solvmanifolds, as follows.

TheOREM 3.3. Let $M=S / \Gamma$ be a solvmanifold and let $\iota: \mathbb{G} \rightarrow G L(V)$ be a rational representation of a complex, semisimple linear algebraic group of rank 1 , with tangent map $\theta: \mathfrak{s l}_{2} \rightarrow \mathfrak{g l}(V)$. Then the germ at 1 of $\operatorname{Hom}(\Gamma, \mathbb{G})$ is isomorphic to the germ at 0 of the cone on $\mathbb{P}\left(H^{1}(M)\right) \times \mathbb{P}\left(\mathfrak{s l}_{2}\right)$, and the embedded topological Green-Lazarsfeld germs are given by

$$
\mathscr{V}_{1}^{i}(M, \iota)_{(1)}= \begin{cases}\emptyset & \text { if } H^{i}(M)=0 \\ \operatorname{cone}\left(\mathbb{P}\left(H^{1}(M)\right) \times V(\operatorname{det} \circ \theta)\right)_{(0)} & \text { otherwise. }\end{cases}
$$


Proof. Let $A$ be a Kasuya model for $M$. Theorem B from [5] allows us to replace $\operatorname{Hom}(\Gamma, \mathbb{G})_{(1)}$ by $\mathscr{F}\left(A, \mathfrak{s l}_{2}\right)_{(0)}$ and $\mathscr{V}_{1}^{i}(M, \iota)_{(1)}$ by $\mathscr{R}_{1}^{i}(A, \theta)_{(0)}$. The assertion about $\operatorname{Hom}(\Gamma, \mathbb{G})_{(1)}$ follows then from Lemma 3.1 and the description $(6)$ of $\mathscr{F}^{1}\left(A, \mathfrak{s l}_{2}\right)$, since $H^{\bullet}(A) \simeq H^{\bullet}(M)$. If $H^{i}(A)=0, \mathscr{R}_{1}^{i}(A, \theta)_{(0)}=\emptyset$, by $(3)$. When $H^{i}(A) \neq 0$, Lemma 3.2 and Corollary 2.5 together imply that $\mathscr{R}_{1}^{i}(A, \theta)_{(0)}=\Pi(A, \theta)_{(0)}$. The identification of $\Pi(A, \theta)$ with the cone on $\mathbb{P}\left(H^{1}(M)\right) \times V(\operatorname{det} \circ \theta)$ follows as before.

Note that the complete description of embedded non-abelian jump loci from Theorem 3.3 depends only on $\theta$ and the untwisted Betti numbers of $M$, and extends the similar result on nilmanifolds obtained in $[\mathbf{1 8}]$.

EXAmPle 3.4. When the Lie group $S$ is nilpotent, a remarkable result of Dixmier [8] says that the untwisted Betti numbers of $M$ have no gaps, i.e., $b_{i}(M) \neq 0$ for all $i \leq \operatorname{dim} M$. This no longer holds for solvmanifolds. Indeed, Kasuya constructed in $[\mathbf{1 4}$, Example 10.4], for every $s \geq 1$, a solvmanifold $M$ of dimension $2 s+2$ with the property that $b_{s+1}(M)=0$.

We close this section by examining the rank one jump loci of virtually polycyclic groups. Let $M$ be a connected $\mathrm{CW}$-complex with finite 1-skeleton and (finitely generated) fundamental group $\pi$. Denote by $\mathbb{T}(M):=\operatorname{Hom}\left(\pi, \mathbb{C}^{\times}\right)=\operatorname{Hom}\left(\pi_{\mathrm{ab}}, \mathbb{C}^{\times}\right)$the affine character torus, corresponding to the case $\iota=\mathrm{id}_{\mathbb{C}^{\times}}$. For $\rho \in \mathbb{T}(M)$, let $\mathbb{C}_{\rho}$ be the associated rank 1 local system on $M$, identified with the right $\pi$-module $\mathbb{C}_{\rho}$, where $z \cdot g=z \rho(g)$, for $z \in \mathbb{C}$ and $g \in \pi$. As noted in [18], the rank 1 jump loci (the characteristic varieties $\left.\mathscr{V}_{r}^{i}(M)\right)$ are given by

$$
\mathscr{V}_{r}^{i}(M)=\left\{\rho \in \mathbb{T}(M) \mid \operatorname{dim} H_{i}\left(M, \mathbb{C}_{\rho}\right) \geq r\right\} .
$$

When $M$ is aspherical, we will replace it by $\pi$ in the notation. Our goal is to prove the following.

THEOREM 3.5. If $\pi$ is a virtually polycyclic group, then the characteristic variety $\mathscr{V}_{1}^{i}(\pi)$ is finite, for all $i$.

This extends a result from [17], where it was shown that $\bigcup_{i \geq 0} \mathscr{V}_{1}^{i}(\pi)=\{1\}$, when $\pi$ is a finitely generated nilpotent group. At the same time, this gives a more precise version of Lemma 3.2, when $M=S / \Gamma$ is a solvmanifold (aspherical, with polycyclic fundamental group $\Gamma$ ) with cdga model $A$, since $\mathscr{V}_{r}^{i}(M)_{(1)} \simeq \mathscr{R}_{r}^{i}(A)_{(0)}$, by the main result of [5]. Theorem 3.5 was proved for solv-lattices by Kasuya in [15], using a different method.

We begin by pointing out a useful virtual property, for characteristic varieties of groups.

Lemma 3.6. Let $f: K \hookrightarrow G$ be the inclusion of a finite index subgroup $K$ in a finitely generated group $G$. Then $f^{*}: \mathbb{T}(G) \rightarrow \mathbb{T}(K)$ has finite fibers and sends $\mathscr{V}_{r}^{i}(G)$ into $\mathscr{V}_{r}^{i}(K)$, for all $i, r$. In particular, if $\mathscr{V}_{1}^{i}(K)$ is finite for all $i$, then $G$ has the same property. 
Proof. Clearly, $f_{\mathrm{ab}}: K_{\mathrm{ab}} \rightarrow G_{\mathrm{ab}}$ has finite index image. By the exactness of the $\mathbb{T}$-functor, $f^{*}$ has finite kernel, which proves the first assertion. For $\rho \in \mathbb{T}(G)$, a transfer argument (see $\left[\mathbf{2}\right.$, III.9]) shows that $f_{\bullet}: H_{\bullet}\left(K, \mathbb{C}_{f^{*} \rho}\right) \rightarrow H_{\bullet}\left(G, \mathbb{C}_{\rho}\right)$ is surjective, whence our second claim. The last property follows from the first two.

If $\pi$ is virtually polycyclic, it contains a finite index subgroup $\pi_{0}$ which is polycyclic with infinite cyclic quotients in a composition series, by [25, Lemma 4.6]. Due to Lemma 3.6, we may thus assume in Theorem 3.5 that $\pi$ is actually poly- $\mathbb{Z}$, and argue by induction on the length of a composition series.

The induction step goes as follows. Let $\alpha$ be an automorphism of a finitely generated group $G$. Denote by $G_{\alpha}$ the semidirect product $G \rtimes_{\alpha} \mathbb{Z}$ and consider the exact sequence

$$
1 \rightarrow G \stackrel{j}{\longrightarrow} G_{\alpha} \stackrel{p}{\longrightarrow} \mathbb{Z} \rightarrow 1
$$

We are going to use, for a $G_{\alpha}$-module $U$, the Hochschild-Serre spectral sequence of the group extension (9) ([2, p. 171]),

$$
E_{s t}^{2}=H_{s}\left(\mathbb{Z}, H_{t}(G, U)\right) \Longrightarrow H_{s+t}\left(G_{\alpha}, U\right),
$$

which collapses at the $E^{2}$-term. We denote by $\tau=\sigma(1) \in G_{\alpha}$ the lift of $1 \in \mathbb{Z}$ to $G_{\alpha}$, via the canonical section $\sigma$ of $p$.

We start by using trivial coefficients $\mathbb{Z}$, to describe character tori. We find that $\left(G_{\alpha}\right)_{\mathrm{ab}} \simeq C \oplus \mathbb{Z}$, where $C$ denotes the coinvariants of $\alpha_{\mathrm{ab}}$ acting on $G_{\mathrm{ab}}$. Thus, $\rho \in \mathbb{T}\left(G_{\alpha}\right)$ is identified with $(\chi, \lambda) \in \mathbb{T}(C) \times \mathbb{C}^{\times}, \chi$ is identified with $j^{*} \rho \in \mathbb{T}(G)$, and $\lambda=\rho(\tau)$.

For $U=\mathbb{C}_{\rho}$, the spectral sequence (10) collapses to the isomorphism

$$
H_{i}\left(G_{\alpha}, \mathbb{C}_{\rho}\right)=H_{0}\left(\mathbb{Z}, H_{i}\left(G, \mathbb{C}_{\chi}\right)\right) \oplus H_{1}\left(\mathbb{Z}, H_{i-1}\left(G, \mathbb{C}_{\chi}\right)\right) .
$$

Moreover, the action of $1 \in \mathbb{Z}$ on $H_{\bullet}\left(G, \mathbb{C}_{\chi}\right)$ may be described as follows, cf. [2, p. 171 and pp. 78-79]. Note that $\alpha: G \rightarrow G$ is conjugation by $\tau$. Let $(\alpha$, id $):\left(G, \mathbb{C}_{\chi}\right) \rightarrow\left(G, \mathbb{C}_{\chi}\right)$ be the automorphism in the category of coefficients associated to $\alpha$ and $\operatorname{id}_{\mathbb{C}}$. Then the right action of $1 \in \mathbb{Z}$ on $H \bullet\left(G, \mathbb{C}_{\chi}\right)$ is given by $\lambda \cdot(\alpha, \mathrm{id})_{*}^{-1}$.

LEMma 3.7. If $\operatorname{dim} H_{i}(G, U)<\infty$ for all $i$ and every finite-dimensional $G$-module $U$, then the same property holds for $G_{\alpha}$.

Proof. This is a straightforward consequence of the spectral sequence (10).

LEMma 3.8. Assume that $G$ has the finiteness property from Lemma 3.7. In the above setting, $\rho \in \mathscr{V}_{1}^{i}\left(G_{\alpha}\right)$ if and only if either $j^{*} \rho=\chi \in \mathscr{V}_{1}^{i}(G)$ and $\lambda$ is an eigenvalue of $(\alpha, \mathrm{id})_{* i}: H_{i}\left(G, \mathbb{C}_{\chi}\right) \rightarrow H_{i}\left(G, \mathbb{C}_{\chi}\right)$ or the same conditions hold for $i-1$.

Proof. By definition (8), $\rho \in \mathscr{V}_{1}^{i}\left(G_{\alpha}\right)$ if and only if $H_{i}\left(G_{\alpha}, \mathbb{C}_{\rho}\right) \neq 0$. We infer from (11) that $H_{i}\left(G_{\alpha}, \mathbb{C}_{\rho}\right) \neq 0$ if and only if 1 is an eigenvalue for the monodromy action of $1 \in \mathbb{Z}$ on either $H_{i}\left(G, \mathbb{C}_{\chi}\right)$ or $H_{i-1}\left(G, \mathbb{C}_{\chi}\right)$. Our claim follows.

Proof of Theorem 3.5. As noticed before, we may assume that $\pi$ is poly- $\mathbb{Z}$. We suppose inductively that the group $G$ from (9) has the finiteness property from 
Lemma 3.7, and Theorem 3.5 holds for $G$. We will finish the induction by showing that both properties are inherited by $G_{\alpha}$. Lemma 3.7 takes care of the first property. We may use then Lemma 3.8 to deduce that, if $\rho \in \mathscr{V}_{1}^{i}\left(G_{\alpha}\right)$, then $\chi=j^{*} \rho$ belongs to the finite set $\mathscr{V}_{1}^{i}(G) \cup \mathscr{V}_{1}^{i-1}(G)$, and the second component of $\rho, \lambda$, is among the finitely many eigenvalues of $(\alpha, \mathrm{id})_{* q}$ acting on $H_{q}\left(G, \mathbb{C}_{\chi}\right)$, for $q=i$ or $i-1$. This completes our proof.

\section{Lie algebras.}

The tools developed in Section 2 also enable us to describe completely the germs at 0 of both $\mathfrak{s l}_{2}$-valued flat connections and their depth 1 resonance subvarieties, for cochain cdga's of finite-dimensional Lie algebras.

By a result of Hattori $[\mathbf{1 1}]$, the $\operatorname{cdga} A^{\bullet}=\mathcal{C}^{\bullet}(\mathfrak{s} \otimes \mathbb{C})$ is a model for the solvmanifold $S / \Gamma$, when the Lie algebra $\mathfrak{s}$ of $S$ is completely solvable. Due to Lemmas 3.1 and 3.2, the comparison theorem with the corresponding topological germs at 1 from [5] implies that $\mathscr{F}\left(A, \mathfrak{s l}_{2}\right)_{(0)}=\mathscr{F}^{1}\left(A, \mathfrak{s l}_{2}\right)_{(0)}$ and $A$ has trivial rank one resonance in degree $i$, if $H^{i}(A) \neq 0$. Therefore, Corollary 2.5 may be used for the cochain cdga $A$ and $\mathfrak{g}=\mathfrak{s l}_{2}$. In what follows, we aim at extending this approach to an arbitrary cochain cdga $A=\mathcal{C} \bullet \mathfrak{h}$, assuming only that the Lie algebra $\mathfrak{h}$ is finite-dimensional.

REMARK 4.1. Millionschikov proved in [19] that $\bigcup_{t>0} \mathscr{R}_{1}^{t}\left(\mathcal{C}^{\bullet} \mathfrak{s}\right)$ is finite, for an arbitrary solvable Lie algebra $\mathfrak{s}$. By the above discussion, this is an infinitesimal analogue of Theorem 3.5, in the completely solvable case.

We refer the reader to $[\mathbf{1 2}$, Chapters VII-VIII $]$ for basic facts related to Lie algebra (co)homology. Let $\mathfrak{h}$ be a finite-dimensional Lie algebra. Note that $\mathcal{C} \bullet \mathfrak{h}=\left(\bigwedge^{\bullet} \mathfrak{h}^{*}, d\right)$ has the exterior algebra on the dual vector space $\mathfrak{h}^{*}$ as underlying commutative graded algebra, hence $\mathcal{C}^{\bullet} \mathfrak{h}$ is connected and finite-dimensional. Denoting by $H^{\bullet}(\mathfrak{h}, U)$ the Lie cohomology of $\mathfrak{h}$ with coefficients in the $\mathfrak{h}$-module $U$, note also that $H^{\bullet}(\mathcal{C h})$ is the Lie cohomology of $\mathfrak{h}$ with trivial $\mathbb{C}$-coefficients, denoted simply by $H^{\bullet}(\mathfrak{h})$. Hence, $\mathscr{F}(\mathcal{C} \bullet \mathfrak{h})=$ $H^{1}(\mathfrak{h})$.

We will need a couple of results on jump loci of cochain cdga's from [18]. First, for any finite-dimensional Lie algebra $\mathfrak{g}$, there is a natural isomorphism between the variety $\mathscr{F}\left(\mathcal{C}^{\bullet} \mathfrak{h}, \mathfrak{g}\right)$ and the Lie representation variety $\operatorname{Rep}(\mathfrak{h}, \mathfrak{g}) \subseteq \mathfrak{h}^{*} \otimes \mathfrak{g}=\operatorname{Hom}(\mathfrak{h}, \mathfrak{g})$, consisting of all Lie homomorphisms from $\mathfrak{h}$ to $\mathfrak{g}$. Moreover, $\mathscr{F}^{1}\left(\mathcal{C}^{\bullet} \mathfrak{h}, \mathfrak{g}\right)$ is identified with $\operatorname{Rep}^{1}(\mathfrak{h}, \mathfrak{g}):=\{\varphi \in \operatorname{Rep}(\mathfrak{h}, \mathfrak{g}) \mid \operatorname{rank}(\varphi) \leq 1\}$. For $\mathfrak{g}=\mathbb{C}$ (the rank one case), clearly $\operatorname{Rep}(\mathfrak{h}, \mathbb{C})=\operatorname{Rep}\left(\mathfrak{h}_{\mathrm{ab}}, \mathbb{C}\right)=\operatorname{Hom}(\mathfrak{h} /[\mathfrak{h}, \mathfrak{h}], \mathbb{C})=\operatorname{Hom}\left(H_{1}(\mathfrak{h}), \mathbb{C}\right)=H^{1}(\mathfrak{h})$, thus recovering the previous identification. For $\omega \in H^{1}(\mathfrak{h})$, we will denote by $\omega \mathbb{C}$ the corresponding rank $1 \mathfrak{h}$-module. This leads to the following description of rank 1 resonance:

$$
\mathscr{R}_{r}^{i}\left(\mathcal{C}^{\bullet} \mathfrak{h}\right)=\left\{\omega \in H^{1}(\mathfrak{h}) \mid \operatorname{dim} H^{i}(\mathfrak{h}, \omega \mathbb{C}) \geq r\right\}
$$

We may now establish the Lie analogue of Theorem 3.5, in full generality.

Proposition 4.2. If the Lie algebra $\mathfrak{h}$ is finite-dimensional, then $\mathscr{R}_{1}^{i}\left(\mathcal{C}^{\bullet} \mathfrak{h}\right)$ is finite, for all $i$. In particular, the cdga $\mathcal{C}^{\bullet} \mathfrak{h}$ has trivial resonance in degree $i$, if $H^{i}(\mathfrak{h}) \neq 0$. 
Proof. By Levi's theorem [12, p. 250], $\mathfrak{h}=\mathfrak{s} \rtimes \mathfrak{g}$, with $\mathfrak{s}$ solvable and $\mathfrak{g}$ semisimple. Consider the Lie extension $0 \rightarrow \mathfrak{s} \stackrel{j}{\longrightarrow} \mathfrak{h} \longrightarrow \mathfrak{g} \rightarrow 0$, and the associated natural exact sequence [12, p. 238]

$$
H_{2}(\mathfrak{h}) \rightarrow H_{2}(\mathfrak{g}) \rightarrow \mathfrak{s} /[\mathfrak{h}, \mathfrak{s}] \rightarrow H_{1}(\mathfrak{h}) \rightarrow H_{1}(\mathfrak{g}) \rightarrow 0,
$$

where $H_{1}(\mathfrak{g})$ and $H_{2}(\mathfrak{g})$ vanish by semisimplicity, cf. [12, p. 247 and p. 249]. We infer that the map induced on abelianizations, $j_{\mathrm{ab}}: H_{1}(\mathfrak{s}) \rightarrow H_{1}(\mathfrak{h})$, may be identified with the surjection $\mathfrak{s} /[\mathfrak{s}, \mathfrak{s}] \rightarrow \mathfrak{s} /[\mathfrak{h}, \mathfrak{s}]$; in particular, $j^{*}: H^{1}(\mathfrak{h}) \hookrightarrow H^{1}(\mathfrak{s})$ is injective.

For $\omega \in H^{1}(\mathfrak{h})$, the extension gives rise to the Grothendieck-Hochschild-Serre spectral sequence $[\mathbf{1 2}$, p. 305]

$$
H^{s}\left(\mathfrak{g}, H^{t}\left(\mathfrak{s}, j^{*} \omega \mathbb{C}\right)\right) \Longrightarrow H^{s+t}(\mathfrak{h}, \omega \mathbb{C}) .
$$

If $j^{*} \omega \notin \bigcup_{t>0} \mathscr{R}_{1}^{t}\left(\mathcal{C}^{\bullet} \mathfrak{s}\right),(14)$ implies by (12) that $H^{\bullet}(\mathfrak{h}, \omega \mathbb{C})=0$. On the other hand, the solvability of $\mathfrak{s}$ forces $\bigcup_{t>0} \mathscr{R}_{1}^{t}\left(\mathcal{C}^{\bullet} \mathfrak{s}\right)$ to be finite, as shown by Millionschikov in [19]. Our claim follows.

We know from $[\mathbf{1 8}]$ that $\mathscr{F}\left(\mathcal{C}^{\bullet} \mathfrak{h}, \mathfrak{s l}_{2}\right)=\mathscr{F}^{1}\left(\mathcal{C}^{\bullet} \mathfrak{h}, \mathfrak{s l}_{2}\right)$, when $\mathfrak{h}$ is nilpotent. Now, we have to prove that $\mathscr{F}\left(\mathcal{C}^{\bullet} \mathfrak{h}, \mathfrak{s l}_{2}\right)_{(0)}=\mathscr{F}^{1}\left(\mathcal{C} \bullet \mathfrak{h}, \mathfrak{s l}_{2}\right)_{(0)}$, in general. We will use the standard basis of $\mathfrak{s l}_{2},\left\{H, X_{+}, X_{-}\right\}$(where $H$ is diagonal with $\operatorname{Eigen}(H)=\{ \pm 1\}$ ), for which $\left[X_{+}, X_{-}\right]=H$ and $\left[H, X_{\epsilon}\right]=2 \epsilon X_{\epsilon}$.

We start by analyzing a simple metabelian case: $\mathfrak{h}=V \rtimes_{\alpha} \mathbb{C}$, where $V$ is abelian and $\alpha \in \mathfrak{g l}(V)$. It is easy to check that $\mathfrak{h}$ is non-nilpotent if and only if $\alpha$ has a nonzero eigenvalue. Choose a basis $\left\{z_{i}\right\}$ of $V$ for which $\alpha$ is in Jordan normal form. For an eigenvalue $\lambda \in \operatorname{Eigen}(\alpha)$, and an $r$-Jordan block of type $\lambda, V(\lambda), \alpha z_{1}=\lambda z_{1}$ and $\alpha z_{i}=\lambda z_{i}+z_{i-1}$ for $1<i \leq r$. We will use the basis $\left\{z_{i}, u\right\}$ for $\mathfrak{h}$, where $u$ corresponds to $1 \in \mathbb{C}$.

For $\varphi \in \operatorname{Rep}\left(\mathfrak{h}, \mathfrak{s l}_{2}\right)$, we know that, on $V, \varphi z_{i}=t_{i} Z$ with $t_{i} \in \mathbb{C}$, for some $Z \in \mathfrak{s l}_{2}$, since $V$ is abelian. We set $\varphi u=U \in \mathfrak{s l}_{2}$. When $\mathfrak{h}$ is not nilpotent, we denote by $f$ the regular function on $\operatorname{Hom}\left(\mathfrak{h}, \mathfrak{s l}_{2}\right)$ defined by $f(\varphi)=\prod\left(\operatorname{det} U+\lambda^{2} / 4\right)$, where the product is taken over the non-zero eigenvalues $\lambda \in \operatorname{Eigen}(\alpha)$. Clearly, $f(0) \neq 0$.

Lemma 4.3. For $\mathfrak{h}=V \rtimes_{\alpha} \mathbb{C}$ as above, the following hold.

(1) Assuming that $\mathfrak{h}$ is non-nilpotent, $\varphi \in \operatorname{Rep}\left(\mathfrak{h}, \mathfrak{s l}_{2}\right) \backslash \operatorname{Rep}^{1}\left(\mathfrak{h}, \mathfrak{s l}_{2}\right)$ if and only if there is $0 \neq \lambda \in \operatorname{Eigen}(\alpha)$ and $\epsilon \in\{ \pm 1\}$ such that $\varphi$ has the following form (up to $G L_{2}$-conjugation):

- $\varphi u=\lambda / 2 \epsilon H$;

- $\varphi=0$, on each Jordan block $V\left(\lambda^{\prime}\right)$ with $\lambda^{\prime} \neq \lambda$;

- on each $r$-Jordan block $V(\lambda), \varphi z_{i}=t_{i} X_{\epsilon}$ for $1 \leq i \leq r$, with $t_{i}=0$ for $i<r$;

- $t_{r} \neq 0$, for at least one such block $V(\lambda)$.

Furthermore, in this situation $f(\varphi)=0$.

(2) $\operatorname{Rep}\left(\mathfrak{h}, \mathfrak{s l}_{2}\right)=\operatorname{Rep}^{1}\left(\mathfrak{h}, \mathfrak{s l}_{2}\right)$ if and only if $\mathfrak{h}$ is nilpotent. 
Proof. Part (1). The rank condition on $\varphi$ translates to the fact that $\operatorname{rank}\{U, Z\}=$ 2 plus the property that the vector $\underline{t}=\left(t_{1}, \ldots, t_{r}\right)$ is non-zero for at least one Jordan block. Clearly, $\varphi \in \operatorname{Rep}\left(\mathfrak{h}, \mathfrak{s l}_{2}\right)$ if and only if, for every $r$-Jordan block $V(\lambda), \varphi \alpha z_{i}=$ $\varphi\left[u, z_{i}\right]=t_{i}[U, Z]$, for $1 \leq i \leq r$. In more detail, this means that

$$
\lambda t_{1} Z=t_{1}[U, Z] \quad \text { and } \quad\left(\lambda t_{i}+t_{i-1}\right) Z=t_{i}[U, Z], \quad \text { for } \quad 1<i \leq r .
$$

When $\underline{t} \neq 0$, (15) may be solved as follows. Let $i$ be the first value for which $t_{i} \neq 0$. We deduce from (15) that $[U, Z]=\lambda Z$. Since $Z \neq 0$, it follows that (15) is equivalent to $t_{i}=0$ for $1 \leq i<r$.

We are left with solving the equation $[U, Z]=\lambda Z$, which implies that $\lambda \neq 0$, since otherwise our assumption $\operatorname{rank}\{U, Z\}=2$ would be violated. This in turn forces $\operatorname{det} U \neq 0$. Indeed, otherwise $U$ would be nilpotent, hence ad-nilpotent, which implies $\lambda=0$. Thus, we may assume that $U=t H$ with $t \in \mathbb{C}^{\times}$, modulo $G L_{2}$-conjugation. This implies that we may also assume $Z=X_{\epsilon}$ for some $\epsilon \in\{ \pm 1\}$ and $t=\lambda / 2 \epsilon$. Plainly, $\left[t H, X_{\epsilon}\right]=\lambda X_{\epsilon}$.

This completes our explicit description of $\operatorname{Rep}\left(\mathfrak{h}, \mathfrak{s l}_{2}\right) \backslash \operatorname{Rep}^{1}\left(\mathfrak{h}, \mathfrak{s l}_{2}\right)$. The property $f(\varphi)=0$ becomes a direct consequence of the construction of $f$, since $\operatorname{det} H=-1$.

Part (2). If $\mathfrak{h}$ is nilpotent, the claim is proved in [18]. If $\mathfrak{h}$ is non-nilpotent, then $\operatorname{Rep}\left(\mathfrak{h}, \mathfrak{s l}_{2}\right) \neq \operatorname{Rep}^{1}\left(\mathfrak{h}, \mathfrak{s l}_{2}\right)$, by Part (1).

At the next step, we settle the solvable case.

LEMma 4.4. Let $\mathfrak{s}$ be a finite-dimensional solvable Lie algebra. There is a Zariski closed subset $W \subseteq \operatorname{Hom}\left(\mathfrak{s}, \mathfrak{s l}_{2}\right)$ not containing 0 such that $\operatorname{Rep}\left(\mathfrak{s}, \mathfrak{s l}_{2}\right) \subseteq \operatorname{Rep}^{1}\left(\mathfrak{s}, \mathfrak{s l}_{2}\right) \cup W$. In particular, $\operatorname{Rep}\left(\mathfrak{s}, \mathfrak{s l}_{2}\right)_{(0)}=\operatorname{Rep}^{1}\left(\mathfrak{s}, \mathfrak{s l}_{2}\right)_{(0)}$.

Proof. We argue by induction on the length of the derived series, $\left\{\mathfrak{s}^{(k)}\right\}$. If $\mathfrak{s}^{(1)}=$ $\mathfrak{s}^{\prime}=0$, then $\mathfrak{s}$ is nilpotent and we may take $W=\emptyset$. For the inductive step, we assume that $\mathfrak{s}^{(k+1)}=0$ and we consider the Lie extension $0 \rightarrow V \longrightarrow \mathfrak{s} \stackrel{p}{\longrightarrow} \mathfrak{h} \rightarrow 0$, where $V=\mathfrak{s}^{(k)}$ is abelian and $\mathfrak{h}=\mathfrak{s} / \mathfrak{s}^{(k)}$ satisfies $\mathfrak{h}^{(k)}=0$. Hence, $\operatorname{Rep}\left(\mathfrak{h}, \mathfrak{s l}_{2}\right) \subseteq \operatorname{Rep}^{1}\left(\mathfrak{h}, \mathfrak{s l}_{2}\right) \cup W^{\prime}$, with $W^{\prime} \subseteq \operatorname{Hom}\left(\mathfrak{h}, \mathfrak{s l}_{2}\right)$ Zariski closed and $0 \notin W^{\prime}$. Clearly, $p^{*} W^{\prime} \subseteq \operatorname{Hom}\left(\mathfrak{s}, \mathfrak{s l}_{2}\right)$ is Zariski closed and $0 \notin p^{*} W^{\prime}$.

Let $\left\{u_{i}\right\} \subseteq \mathfrak{s}$ be a lift of an $\mathfrak{h}$-basis. Plainly, for each $i, \mathfrak{h}_{i}:=V \oplus \mathbb{C} \cdot u_{i}$ is a Lie subalgebra of $\mathfrak{s}$, and $\mathfrak{h}_{i}=V \rtimes_{\alpha_{i}} \mathbb{C}$, where $\alpha_{i}=\operatorname{ad}_{V}\left(u_{i}\right)$. If $\mathfrak{h}_{i}$ is nilpotent for all $i$, we claim that $\operatorname{Rep}\left(\mathfrak{s}, \mathfrak{s l}_{2}\right) \subseteq \operatorname{Rep}^{1}\left(\mathfrak{s}, \mathfrak{s l}_{2}\right) \cup p^{*} \operatorname{Rep}\left(\mathfrak{h}, \mathfrak{s l}_{2}\right)$. Consequently, we are done by induction, since clearly $p^{*} \operatorname{Rep}^{1}\left(\mathfrak{h}, \mathfrak{s l}_{2}\right) \subseteq \operatorname{Rep}^{1}\left(\mathfrak{s}, \mathfrak{s l}_{2}\right)$. To prove the claim, suppose that $\varphi \in \operatorname{Rep}\left(\mathfrak{s}, \mathfrak{s l}_{2}\right) \backslash p^{*} \operatorname{Rep}\left(\mathfrak{h}, \mathfrak{s l}_{2}\right)$. This implies that $\varphi(V)$ is 1-dimensional, since $V$ is abelian. By our nilpotence assumption, we infer that $\varphi u_{i} \in \varphi(V)$ for all $i$, whence $\varphi \in \operatorname{Rep}^{1}\left(\mathfrak{s}, \mathfrak{s l}_{2}\right)$, as claimed.

Thus, we may assume that $\mathfrak{h}_{i}$ is non-nilpotent, for some $i$. For each such $i$, let $f_{i}$ be a regular lift to $\operatorname{Hom}\left(\mathfrak{s}, \mathfrak{s l}_{2}\right)$ of the polynomial function $f$ on $\operatorname{Hom}\left(\mathfrak{h}_{i}, \mathfrak{s l}_{2}\right)$ from Lemma 4.3(1). Let $V(F) \subseteq \operatorname{Hom}\left(\mathfrak{s}, \mathfrak{s l}_{2}\right)$ be the zero set of $F:=\prod f_{i}$, which clearly does not contain 0 . We claim that we may take $W=p^{*} W^{\prime} \cup V(F)$.

Indeed, let $\varphi \in \operatorname{Rep}\left(\mathfrak{s}, \mathfrak{s l}_{2}\right) \backslash \operatorname{Rep}^{1}\left(\mathfrak{s}, \mathfrak{s l}_{2}\right)$ be arbitrary. If $\varphi \in p^{*} \operatorname{Rep}\left(\mathfrak{h}, \mathfrak{s l}_{2}\right) \subseteq$ $p^{*} \operatorname{Rep}^{1}\left(\mathfrak{h}, \mathfrak{s l}_{2}\right) \cup p^{*} W^{\prime}$, we are done. If $\varphi \notin p^{*} \operatorname{Rep}\left(\mathfrak{h}, \mathfrak{s l}_{2}\right)$, pick $i$ such that $\varphi_{i} \notin$ 
$\operatorname{Rep}^{1}\left(\mathfrak{h}_{i}, \mathfrak{s l}_{2}\right)$, where $\varphi_{i}$ denotes the restriction of $\varphi$ to $\mathfrak{h}_{i}$, using an argument as before. In particular, $\mathfrak{h}_{i}$ is non-nilpotent. By Lemma $4.3(1), f_{i}(\varphi)=0$. Therefore, $F(\varphi)=0$, which completes our proof.

Now, we describe a procedure that reduces the study of germs at 0 of arbitrary Lie representation varieties to the case when the domain is solvable. We begin with the semisimple case.

LEMma 4.5. Let $\mathfrak{g}$ be a semisimple and $\mathfrak{k}$ be an arbitrary finite-dimensional Lie algebra. Then $\operatorname{Rep}(\mathfrak{g}, \mathfrak{k})_{(0)}=\{0\}$.

Proof. By Ado's theorem, $\mathfrak{k}$ may be embedded in $\mathfrak{g l}(V)$, for some finitedimensional vector space $V$. Note that $G L(V)$ acts by conjugation on $\operatorname{Rep}(\mathfrak{g}, \mathfrak{g l}(V))$, and the $G L(V)$-orbit of 0 is $\{0\}$. By a classical result of Nijenhuis and Richardson [20, p. 3], $\operatorname{Rep}(\mathfrak{g}, \mathfrak{g l}(V))$ is the union of finitely many Zariski closed $G L(V)$ orbits, provided $\mathfrak{g}$ is semisimple. (This is due to the fact that $H^{1}(\mathfrak{g}, U)=0$ when $\operatorname{dim} U<\infty$, see $[\mathbf{1 2}$, p. 247].) Our claim follows then from the obvious equality $\operatorname{Rep}(\mathfrak{g}, \mathfrak{k})=\operatorname{Rep}(\mathfrak{g}, \mathfrak{g l}(V)) \cap \operatorname{Hom}(\mathfrak{g}, \mathfrak{k})$.

For an arbitrary finite-dimensional Lie algebra $\mathfrak{h}$, pick a Levi decomposition $\mathfrak{h}=$ $\mathfrak{s} \rtimes_{\alpha} \mathfrak{g}$, with $\mathfrak{s}$ solvable, $\mathfrak{g}$ semisimple and $\alpha$ a Lie homomorphism from $\mathfrak{g}$ to the Lie algebra of derivations, $\operatorname{Der}(\mathfrak{s}) \subseteq \mathfrak{g l}(\mathfrak{s})$. Let $\mathfrak{k}$ be another finite-dimensional Lie algebra. The semi-direct product structure of $\mathfrak{h}$ implies that the map

$$
\varphi \in \operatorname{Hom}(\mathfrak{h}, \mathfrak{k}) \mapsto\left(\Phi:=\varphi_{\mid \mathfrak{s}}, \Psi:=\varphi_{\mid \mathfrak{g}}\right) \in \operatorname{Hom}(\mathfrak{s}, \mathfrak{k}) \times \operatorname{Hom}(\mathfrak{g}, \mathfrak{k})
$$

identifies the variety $\operatorname{Rep}(\mathfrak{h}, \mathfrak{k})$ with the Zariski closed subset of $\operatorname{Rep}(\mathfrak{s}, \mathfrak{k}) \times \operatorname{Rep}(\mathfrak{g}, \mathfrak{k})$ given by the equations

$$
\Phi(\alpha(y) x)=[\Psi y, \Phi x], \quad \text { for } \quad x \in \mathfrak{s} \quad \text { and } \quad y \in \mathfrak{g} .
$$

Denote by $\widetilde{\mathfrak{s}}$ the (solvable) quotient of $\mathfrak{s}$ by the Lie ideal generated by $\alpha(y) x$, for $x \in \mathfrak{s}$ and $y \in \mathfrak{g}$. Let $q: \mathfrak{s} \rightarrow \widetilde{\mathfrak{s}}$ be the quotient map. Consider the regular map

$$
Q: \operatorname{Rep}(\widetilde{\mathfrak{s}}, \mathfrak{k}) \hookrightarrow \operatorname{Rep}(\mathfrak{h}, \mathfrak{k}),
$$

which sends $\widetilde{\Phi}$ to $\left(q^{*} \widetilde{\Phi}, 0\right)$. It follows from $(16)$ that $Q$ identifies the variety $\operatorname{Rep}(\widetilde{\mathfrak{s}}, \mathfrak{k})$ with the Zariski closed subvariety $\operatorname{Rep}(\mathfrak{h}, \mathfrak{k}) \cap \operatorname{Rep}(\mathfrak{s}, \mathfrak{k}) \times 0$.

Proposition 4.6. Let $\mathfrak{h}$ and $\mathfrak{k}$ be finite-dimensional Lie algebras. The map $Q$ from (17) induces an analytic isomorphism $\operatorname{Rep}(\widetilde{\mathfrak{s}}, \mathfrak{k})_{(0)} \simeq \operatorname{Rep}(\mathfrak{h}, \mathfrak{k})_{(0)}$.

Proof. By the above discussion, it is enough to show that, for $(\Phi, \Psi) \in \operatorname{Rep}(\mathfrak{h}, \mathfrak{k})$ near the origin, $\Psi=0$. This follows from Lemma 4.5.

Corollary 4.7. For any finite-dimensional Lie algebra $\mathfrak{h}, \mathscr{F}\left(\mathcal{C} \bullet \mathfrak{h}, \mathfrak{s l}_{2}\right)_{(0)}=$ $\mathscr{F}^{1}\left(\mathcal{C}^{\bullet} \mathfrak{h}, \mathfrak{s l}_{2}\right)_{(0)}$. 
Proof. We have to show that $\operatorname{Rep}(\mathfrak{h}, \mathfrak{k})_{(0)}=\operatorname{Rep}^{1}(\mathfrak{h}, \mathfrak{k})_{(0)}$, when $\mathfrak{k}=\mathfrak{s l}_{2}$. For $\varphi=(\Phi, \Psi) \in \operatorname{Rep}(\mathfrak{h}, \mathfrak{k})$ near 0 , we know from Proposition 4.6 that $\Psi=0$ and $\Phi=$ $q^{*} \widetilde{\Phi}$ with $\widetilde{\Phi} \in \operatorname{Rep}(\widetilde{\mathfrak{s}}, \mathfrak{k})$ near 0 . Lemma 4.4 implies that $\operatorname{dim} \operatorname{im}(\widetilde{\Phi}) \leq 1$, and therefore $\operatorname{dim} \operatorname{im}(\Phi) \leq 1$. We deduce that $\varphi \in \operatorname{Rep}^{1}\left(\mathfrak{h}, \mathfrak{s l}_{2}\right)$, which completes the proof.

We end with the following Lie analog of Theorem 3.3.

THEOREM 4.8. Let $\mathfrak{h}$ be a finite-dimensional complex Lie algebra and let $\theta: \mathfrak{s l}_{2}(\mathbb{C}) \rightarrow \mathfrak{g l}(V)$ be a finite-dimensional Lie representation with $V \neq 0$. Then $\mathscr{F}\left(\mathcal{C} \bullet \mathfrak{h}, \mathfrak{s l}_{2}\right)_{(0)}$ is equal to $\{0\}$ when $H^{1}(\mathfrak{h})=0$, and otherwise is isomorphic to cone $\left(\mathbb{P}\left(H^{1}(\mathfrak{h})\right) \times \mathbb{P}\left(\mathfrak{s l}_{2}\right)\right)_{(0)}$. In the first case, $\mathscr{R}_{1}^{i}\left(\mathcal{C}^{\bullet} \mathfrak{h}, \theta\right)_{(0)}$ is empty or equal to $\{0\}$, depending on whether $H^{i}(\mathfrak{h})$ vanishes or not. In the second case, the embedded germs of depth 1 resonance varieties are given by

$$
\mathscr{R}_{1}^{i}\left(\mathcal{C}^{\bullet} \mathfrak{h}, \theta\right)_{(0)}= \begin{cases}\emptyset & \text { if } H^{i}(\mathfrak{h})=0 \\ \operatorname{cone}\left(\mathbb{P}\left(H^{1}(\mathfrak{h})\right) \times V(\operatorname{det} \circ \theta)\right)_{(0)} & \text { otherwise }\end{cases}
$$

Proof. By Proposition 4.2 and Corollary 4.7, the hypotheses of Corollary 2.5 are satisfied by $A=\mathcal{C}^{\bullet} \mathfrak{h}$ and $\mathfrak{g}=\mathfrak{s l}_{2}$, if $H^{i}(\mathfrak{h}) \neq 0$. The proof goes as in Theorem 3.3, when $H^{1}(\mathfrak{h}) \neq 0$. Finally, assume $H^{1}(\mathfrak{h})=0$. Then $\mathscr{F}\left(\mathcal{C}^{\bullet} \mathfrak{h}, \mathfrak{s l}_{2}\right)_{(0)}=\{0\}$, since $\mathscr{F}^{1}\left(\mathcal{C}^{\bullet} \mathfrak{h}, \mathfrak{s l}_{2}\right)=$ $\{0\}$, by definition (4). The remaining assertions, on $\mathscr{R}_{1}^{i}\left(\mathcal{C}^{\bullet} \mathfrak{h}, \theta\right)_{(0)}$, follow then from (3).

Acknowledgments. We are grateful to the referee, for pertinent remarks and suggestions.

\section{References}

[1] D. Arapura, Geometry of cohomology support loci for local systems I, J. Algebraic Geom., 6 (1997), 563-597.

[ 2 ] K. S. Brown, Cohomology of groups, Grad. Texts in Math., 87, Springer-Verlag, New York-Berlin, 1982.

[ 3 ] A. Dimca, R. Hain and S. Papadima, The abelianization of the Johnson kernel, J. Eur. Math. Soc., 16 (2014), 805-822.

[4] A. Dimca and S. Papadima, Arithmetic group symmetry and finiteness properties of Torelli groups, Ann. of Math. (2), 177 (2013), 395-423.

[5] A. Dimca and S. Papadima, Non-abelian cohomology jump loci from an analytic viewpoint, Commun. Contemp. Math., 16 (2014), 1350025, 47 pp.

[6] A. Dimca, S. Papadima and A. I. Suciu, Topology and geometry of cohomology jump loci, Duke Math. J., 148 (2009), 405-457.

[ 7 ] A. Dimca, S. Papadima and A. Suciu, Algebraic models, Alexander-type invariants, and GreenLazarsfeld sets, Bull. Math. Soc. Sci. Math. Roumanie, 58(106) (2015), 257-269.

[8] J. Dixmier, Cohomologie des algèbres de Lie nilpotentes, Acta Sci. Math. Szeged, 16 (1955), $246-250$.

[9] M. Green and R. Lazarsfeld, Deformation theory, generic vanishing theorems, and some conjectures of Enriques, Catanese and Beauville, Invent. Math., 90 (1987), 389-407.

[10] M. Green and R. Lazarsfeld, Higher obstructions to deforming cohomology groups of line bundles, J. Amer. Math. Soc., 4 (1991), 87-103.

[11] A. Hattori, Spectral sequence in the De Rham cohomology of fibre bundles, J. Fac. Sci. Univ. Tokyo, 8 (1960), 289-331. 
[12] P. J. Hilton and U. Stammbach, A course in homological algebra, Grad. Texts in Math., 4, Springer-Verlag, New York, 1997.

[13] M. Kapovich and J. Millson, On representation varieties of Artin groups, projective arrangements and the fundamental groups of smooth complex algebraic varieties, Inst. Hautes Études Sci. Publ. Math., 88 (1998), 5-95.

[14] H. Kasuya, Minimal models, formality and Hard Lefschetz properties of solvmanifolds with local systems, J. Diff. Geom., 93 (2013), 269-297.

[15] H. Kasuya, Flat bundles and hyper-Hodge decomposition on solvmanifolds, Int. Math. Res. Notices, 19 (2015), 9638-9659.

[16] H. Kasuya, Singularity of the varieties of representations of lattices in solvable Lie groups, J. Topol. Anal., 8 (2016), 273-285.

[17] A. Macinic and S. Papadima, Characteristic varieties of nilpotent groups and applications, In: Proceedings of the Sixth Congress of Romanian Mathematicians (Bucharest, 2007), 1, Romanian Academy, Bucharest, 2009, 57-64.

[18] A. Măcinic, S. Papadima, R. Popescu and A. Suciu, Flat connections and resonance varieties: from rank one to higher ranks, Trans. Amer. Math. Soc., 369 (2017), 1309-1343.

[19] D. V. Millionschikov, Cohomology of solvmanifolds with local coefficients and problems of the Morse-Novikov theory, Russian Math. Surveys, 57 (2002), 813-814.

[20] A. Nijenhuis and R. W. Richardson, Cohomology and deformations in graded Lie algebras, Bull. Amer. Math. Soc., 72 (1966), 1-29.

[21] K. Nomizu, On the cohomology of compact homogeneous spaces of nilpotent Lie groups, Annals of Math., 59 (1954), 531-538.

[22] S. Papadima and A. Suciu, Jump loci in the equivariant spectral sequence, Math. Res. Lett., 21 (2014), 863-883.

[23] S. Papadima and A. Suciu, The Milnor fibration of a hyperplane arrangement: from modular resonance to algebraic monodromy, Proc. London Math. Soc., 114 (2017), 961-1004.

[24] S. Papadima and A. Suciu, Vanishing resonance and representations of Lie algebras, J. Reine Angew. Math., 706 (2015), 83-101.

[25] M. S. Raghunathan, Discrete subgroups of Lie groups, Ergebnisse der Math., 68, Springer-Verlag, New York, 1972.

[26] D. Sullivan, Infinitesimal computations in topology, Inst. Hautes Études Sci. Publ. Math., 47 (1977), 269-331.

\section{Ştefan PAPADIMA}

Simion Stoilow Institute of Mathematics

P.O. Box 1-764

RO-014700 Bucharest, Romania

E-mail: Stefan.Papadima@imar.ro

\section{Laurenţiu PAUnescu}

School of Mathematics and Statistics The University of Sydney

Sydney 2006, Australia

E-mail: laurent@maths.usyd.edu.au 